\title{
ESTUDO EM CLUSTERS TECNOLÓGICOS IBERO AMERICANOS
}

\author{
Fabio Lamartine Barbosa Toledo* \\ fabio.lamartine@brusque.ifc.edu.br
}

\author{
Keller Mafioletti* \\ keller.mafioletti@ifc.edu.br
}

Mohamed Amal*

amal@furb.br

\section{Marianne Hoeltgebaum* \\ profamarianne@gmail.com}

* Universidade Regional de Blumenau - Blumenau, SC / Brasil

\author{
http://dx.doi.org/10.1590/1413-2311.0422015.58412 \\ Recebido em 10/09/2015 \\ Aprovado em 30/05/2016 \\ Disponibilizado em 31/08/2016 \\ Avaliado pelo sistema "double blind review" \\ Revista Eletrônica de Administração \\ Editora-chefe: Aurora Zen \\ ISSN 1413-2311 (versão "on line") \\ Editada pela Escola de Administração da Universidade Federal do Rio Grande do Sul. \\ Periodicidade: Quadrimestral \\ Sistema requerido: Adobe Acrobat Reader
}

\section{RESUMO}

O presente artigo analisa o índice de especialização e sua relação com inovação, internacionalização e a qualificação. Limitando-se a alguns setores inseridos nos clusters tecnológicos das regiões de Florianópolis/SC, Recife/PE, Região do Porto/Portugal e Região da Cataluña/Espanha, buscou-se verificar como o desenvolvimento da inovação (patentes submetidas), a internacionalização (volume de exportação) e a qualificação (mestres e doutores) nestas regiões relaciona-se com o índice de especialização das mesmas. Utilizou-se neste estudo um recorte longitudinal, no qual a evolução do índice de especialização foi analisado ao longo de um período de quatro anos e confrontado com os indicadores: qualificação, internacionalização e inovação. Foram formuladas três hipóteses, as quais foram testadas por meio do tratamento dos dados obtidos nas regiões dos quatro clusters pesquisados.

PALAVRAS CHAVE: Cluster; Inovação; Internacionalização; Qualificação; Polo Tecnológico. 


\begin{abstract}
This article analyzes the specialization index and its relation to innovation, internationalization and qualification. Limited to some sectors inserted in technology clusters in the regions of Florianópolis / SC, Recife / PE, the city of Porto / Portugal and Region of Catalonia / Spain, we tried to see how the development of innovation (patents submitted), internationalization (export volume) and the qualification (master and $\mathrm{PhD}$ ) in these regions is related to the specialization index of the same. Was used in this study a longitudinal cut, in which the evolution of the specialization index was analyzed over a period of four years and faced with the indicators qualification, internationalization and innovation. Three hypotheses were formulated, which were tested by treatment of the data obtained in the four regions surveyed clusters.
\end{abstract}

KEYWORDS: Cluster; Innovation; Internationalization; Qualification; Technological Pole.

\title{
ESTUDIO EN TECNOLÓGICAS CLUSTERS IBERO AMERICANA
}

\begin{abstract}
RESUMEN
El presente artículo analiza el índice de especialización y su relación con la innovación, la internacionalización y calificación. Si uno limitando algunos sectores insertados en tecnológicas clusters establece las Regiones de Florianópolis / SC, Recife / PE , Región de Porto / Portugal y la Región de Cataluña / España, trató si verificar cómo el desarrollo de la innovación (presentado de patentes), internacionalización (el volumen de las exportaciones) y calificación (maestros y doctores) en estas regiones se relaciona con el índice de especialización de la misma. Se utilizó neste estudio una sección longitudinal, no lo que se analizó el índice de especialización evolución junto periodo por cuatro años y se enfrentó con los Indicadores: calificación, internacionalización y la innovación. Fueron tres hipótesis formuladas que se ensayaron a través de la toma y el tratamiento de los datos obtenidos de las regiones cuatro de los conjuntos estudiados.
\end{abstract}

PALABRAS CLAVE: Cluster; Innovación; Internacionalización; Calificación; Polo Tecnológico.

\section{INTRODUÇÃO}

Mercados cada vez mais competitivos e complexos, estagnação de economias internas, instigam a busca de alternativas inteligentes e articuladas entre empresas, governos e instituições, para promoção de novos empreendimentos que tenham potencial para internacionalização, e possam ocupar novos nichos de mercado, promovendo formas de desenvolvimento individuais e coletivas.

Neste cenário, observa-se uma valorização de aglomerações empresariais, sob várias formas, distritos industriais, arranjos produtivos locais, sistemas locais de inovação, neste estudo,

REAd | Porto Alegre - Edição 84 - N 2 - Maio / Agosto 2016 - p. 453 - 476 


\section{Fabio Lamartine Barbosa Toledo, Keller Mafioletti, Mohamed Amal \& Marianne Hoeltgebaum}

admitamos a formação denominada clusters, que segundo Porter (1998, p. 211) "[...] é um agrupamento geograficamente concentrado de empresas inter-relacionadas e instituições correlatas numa determinada área vinculada por elementos comuns e complementares [...]", que permitem aos integrantes uma apropriação de vantagens ditas externalidades como: economia de escala, especialização, fluxo de conhecimento, novos negócios, entre outras.

Isso possibilita um fortalecimento perante as adversidades, atribuindo-lhes diferencial competitivo e benefícios mútuos aliado a possibilidade de maiores ganhos em seus processos produtivos (MARSHAL, 1890; PORTER, 1998; SCHIMITZ, 1999; CASSIOLATTO; LASTRES, 2003).

Entendendo que o impulso econômico alavancado neste tipo de aglomeração visa principalmente atender necessidades externas ao aglomerado, faz com que este tipo de ambiente se torne bastante frutífero a internacionalização, tanto é que vários autores correlacionaram a formação de cluster como determinante ao desempenho exportador, entre eles Becchetti e Rossi (2000); Maccarini, Scabin e Zuchella (2003); Belso-Matinez (2006); Fernhaber, Gilbert e McDougall (2008); Diez-Vial e Fernández-Olmos (2014).

Paralelamente outras pesquisas exploraram como a formação destes conglomerados influenciam na inovação (TRISTÃO et al, 2013; LAI et al, 2014; BAPTISTA; SWANN, 1998; RODRÍGUEZ; VALENCIA, 2008; MATÍNEZ, BELSO-MARTÍNEZ; MAS-VERDÚ, 2012), facilitada pela interação entre os componentes do cluster.

E ainda, o estreitamento do relacionamento entre as empresas, motivado pela clusterização, aumenta a possibilidade do spillover de conhecimento (intencional ou não) (JAFFE, TRANJTENBERG; HENDERSON, 1993), seguindo a perspectiva do conhecimento local, como fator de melhoria econômica regional, outros autores expõe as universidades empreendedoras que ao firmar parceria com empresas, tornam-se elemento de alavanque da economia regional (ETZKOVITZ, 1998).

Nesse contexto, almeja-se, por intermédio da análise dos setores, índices de inovação, grau de especialização, internacionalização e qualificação, entre os clusters tecnológicos de: Florianópolis apoiado pela Fundação CERTI, de Recife alicerçado no Porto Digital, da Região do Porto (Portugal) corroborados pela rede Portuspark e do cluster de tecnologia da informação e comunicação da Cataluña (Espanha) fomentado pelo centro tecnológico Barcelona Digital, responder as seguintes questões:

REAd | Porto Alegre - Edição 84 - Nº 2 - Maio / Agosto 2016 - p. 453 - 476 
- Em que medida o grau de especialização depende do volume de exportações das empresas inseridas nestes clusters?

- Qual o efeito da inovação sobre o grau de especialização destas regiões?

- Quanto maior for o nível de conhecimento do cluster (qualificação profissional) maior será o efeito da inovação sobre a especialização?

Para tanto, o artigo será organizado da seguinte forma: primeiramente apresentação dos fundamentos teóricos, seguido da apresentação das hipóteses, posteriormente uma breve apresentação dos centros de inovação fomentadores dos clusters estudados, logo após apresentação e discussão dos resultados, por fim apresentando as conclusões da pesquisa.

\section{Revisão da Literatura}

Para conceituar cada uma das variáveis utilizadas realizou-se o levantamento de estudos e pesquisas prévias acerca da internacionalização, inovação e o conhecimento do cluster com objetivo de embasar teoricamente este estudo.

O referido suporte teórico do estudo é apresentado na tabela 1, a qual foi elaborada a partir de pesquisa em base de dados com uma seleção de estudos realizados nos últimos quinze anos.

Tabela1 - Estudos que correlacionaram Clusters, Inovação, Internacionalização e Qualificação.

\begin{tabular}{|c|c|c|c|}
\hline Autores & Conteúdo & Indicadores utilizados & Abordagem teórica \\
\hline $\begin{array}{l}\text { Delgado, Porter, } \\
\text { Stern (2014) }\end{array}$ & $\begin{array}{l}\text { O papel do cluster no } \\
\text { desempenho } \\
\text { regional }\end{array}$ & $\begin{array}{l}\text { Especialização, patentes, } \\
\text { crescimento de industrias }\end{array}$ & $\begin{array}{l}\text { Inovação, cluster, } \\
\text { desempenho } \\
\text { industrial }\end{array}$ \\
\hline $\begin{array}{l}\text { Fundeanu e } \\
\text { Badele (2014) }\end{array}$ & $\begin{array}{l}\text { O cluster de inovação como } \\
\text { ferramenta de desenvolvimento } \\
\text { local }\end{array}$ & $\begin{array}{l}\text { Concentração } \\
\text { exploração e desenvolvimento, } \\
\text { qualificação, } \\
\text { existência de organizações } \\
\text { catalisadoras } \\
\text { internacionalização }\end{array}$ & $\begin{array}{l}\text { Tríplice hélice, } \\
\text { modelo quatro } \\
\text { folhas, teorias da } \\
\text { vantagem } \\
\text { competitiva }\end{array}$ \\
\hline $\begin{array}{l}\text { Kafouros et al. } \\
(2008)\end{array}$ & $\begin{array}{l}\text { A influência da } \\
\text { internacionalização na } \\
\text { inovação }\end{array}$ & $\begin{array}{l}\text { Capital Social, intensidade de } \\
\text { P\&D, volume de exportações } \\
\text { da empresa. }\end{array}$ & $\begin{array}{l}\text { Internacionalização, } \\
\text { inovação }\end{array}$ \\
\hline $\begin{array}{l}\text { Libaers e Meyer } \\
\text { (2011) }\end{array}$ & $\begin{array}{l}\text { Inovação, clusters e } \\
\text { internacionalização }\end{array}$ & $\begin{array}{l}\text { Intensidade internacional, } \\
\text { quociente de localização (QL), } \\
\text { número de funcionários, idade } \\
\text { da empresa, patentes } \\
\text { concedidas, experiência } \\
\text { internacional dos gestores }\end{array}$ & $\begin{array}{l}\text { Teoria } \\
\text { dependência } \\
\text { densidade e RBV }\end{array}$ \\
\hline $\begin{array}{l}\text { Guellec e La } \\
\text { Poterie (2001) }\end{array}$ & $\begin{array}{l}\text { Internacionalização baseada } \\
\text { na inovação }\end{array}$ & $\begin{array}{l}\text { Patentes, fatores geográficos e } \\
\text { culturais }\end{array}$ & Teoria das networks \\
\hline
\end{tabular}

REAd | Porto Alegre - Edição 84 - Nº 2 - Maio / Agosto 2016 - p. 453 - 476 


\section{Fabio Lamartine Barbosa Toledo, Keller Mafioletti, Mohamed Amal \& Marianne Hoeltgebaum}

\begin{tabular}{|c|c|c|c|}
\hline Carlsson (2005) & 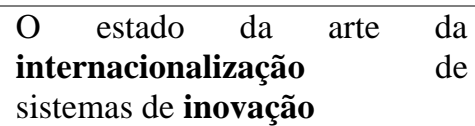 & Artigos científicos & Teoria das networks \\
\hline $\begin{array}{l}\text { Autant-Bernard } \\
\text { et al. (2013) }\end{array}$ & $\begin{array}{l}\text { Papel das políticas regionais de } \\
\text { inovação como apoio as } \\
\text { instituições que geram } \\
\text { conhecimento e aprendizado }\end{array}$ & $\begin{array}{l}\text { Resultados empíricos de } \\
\text { estudos referentes a geografia } \\
\text { de localização e fluxo de } \\
\text { conhecimento localizado ou } \\
\text { externalidades do cluster }\end{array}$ & $\begin{array}{l}\text { Teoria baseada em } \\
\text { recursos }\end{array}$ \\
\hline
\end{tabular}

Continua

Continuação

\begin{tabular}{llrl}
\hline Brennan e & O papel do conhecimento no & $\begin{array}{l}\text { Artigos referentes a tipos de } \\
\text { Garvey (2009) }\end{array}$ & processo \\
internacionalização & conhecimento, natureza do internacionalização, \\
& & $\begin{array}{l}\text { conhecimento e aquisição de born globals, escola } \\
\text { conhecimento nos processos de de uppsala } \\
\text { internacionalização }\end{array}$ \\
& & &
\end{tabular}

\begin{tabular}{lllll}
\hline Wey et al. (2010) & $\begin{array}{l}\text { Difusão da inovação em } \\
\text { clusters }\end{array}$ & $\begin{array}{l}\text { Suportes externos, atributos Redes de cooperação } \\
\text { técnicos }\end{array}$ \\
\hline Breitzman e & $\begin{array}{l}\text { Identificação de tecnologia } \\
\text { emergente (inovação) por meio }\end{array}$ & $\begin{array}{l}\text { Citação de patentes em artigos } \\
\text { científicos, índice de patentes }\end{array}$ \\
Thomasão & de sistemas de patentes & $\begin{array}{l}\text { no cluster, patentes de alto } \\
\text { impacto tecnológico. }\end{array}$ &
\end{tabular}

\begin{tabular}{llll}
\hline $\begin{array}{l}\text { Arcangelis, } \\
\text { Ferri e Padoan } \\
(\mathbf{2 0 0 2 )}\end{array}$ & $\begin{array}{l}\text { Empresas de pequeno porte que } \\
\text { encontram dificuldades da } \\
\text { internacionalização, encontra } \\
\text { saída na aglomeração. }\end{array}$ & Exportações. & Teoria do Cluster \\
\hline Etzkowitz (2012) & $\begin{array}{l}\text { Explora o advento da Tríplice } \\
\text { Hélice na disseminação do } \\
\text { conhecimento e inovação em } \\
\text { cluster tecnológicos }\end{array}$ & $\begin{array}{l}\text { Estudo empírico no MIT, Teoria da Tríplice } \\
\text { Newcastle Universidade de Hélice } \\
\text { com orrelacionando }\end{array}$ \\
& $\begin{array}{l}\text { clusters onde estas } \\
\text { universidades se situam. }\end{array}$ \\
\hline
\end{tabular}

Fonte: Elaborado pelos autores 
Observa-se na tabela que vários autores identificam a inovação e internacionalização como correlatas e fundamentais para o desenvolvimento do cluster. Enfatizam a inovação para a internacionalização do cluster e vice-versa (FONDEANU; BADELE, 2014; KAFOUROS et al., 2008; GUELLEC; LA POTERIE, 2005; WEY et al., 2010). Para estes autores a inovação e internacionalização são interligadas e uma possui influência sobre a outra.

Concomitantemente o conhecimento é apontado como fundamental para o desenvolvimento do cluster e da inovação, esta afirmação é ancorada em estudos de George et al. (2002); Brennan e Garvey (2009); Autant-Bernard et al. (2013); Breitzman e Thomas (2014).

Por fim o papel do cluster na inovação, internacionalização e as externalidades são apontadas nas pesquisas desenvolvidas por Delgado, Porter e Stern (2014), Rodrigues e Valência (2008) e as vantagens da inserção das empresas nos clusters são identificadas nos estudos de Arcangelis et al. (2002); Libaers e Meyer (2011).

\subsection{Internacionalização}

Há basicamente duas abordagens que tratam do processo de internacionalização, a primeira focada no ponto de vista econômico, sendo as escolas defensoras desta abordagem: a escola clássica representada por Smith (1776), Say (1803) e Mill (1848) e a escola neoclássica representada principalmente por Marshall (1890) e Schumpeter (1911).

A segunda abordagem está baseada na questão do comportamento organizacional, sendo que suas principais defensoras são: a Escola de Uppsala (PENROSE, 1962; JOHANSON; VALHNE, 1977), empreendedorismo internacional (ANDERSON, 1997; ZAHRA, 2002), a perspectiva das networks (BJORKMAN; MATSSON, 1988) e ainda a perspectiva visionária (FILION,1991). Para estas teorias, o processo de internacionalização de empresas está intimamente relacionado às características empreendedoras organizacionais.

O modelo da Escola de Uppsala ou Escola Nórdica (JOHANSON; VALHNE, 1977) foi a principal defensora do papel do empreendedor no processo de internacionalização de empresas. Esta escola passou a observar o comportamento organizacional da empresa no processo de internacionalização, e não apenas a questão econômica, até então a única aceita.

De acordo com estudos de Hilal e Hemais (2003), baseados na teoria das networks (evolução do modelo de Uppsala) sugerem que o "[...] grau de internacionalização de uma firma reflete não somente os recursos alocados no exterior, mas também o grau de internacionalização

REAd | Porto Alegre - Edição 84 - Nº 2 - Maio / Agosto 2016 - p. 453 - 476 


\section{Fabio Lamartine Barbosa Toledo, Keller Mafioletti, Mohamed Amal \& Marianne Hoeltgebaum}

da network em que ela está inserida", ou seja, a rede de relacionamentos é fator relevante para o processo de internacionalização das empresas.

Outros estudos têm mostrado o papel de redes de internacionalização de empresas, Johanson e Vahlne (2009) citam as pesquisas de Coviello e Munro (1995,1997) os quais conduziram estudos referente a internacionalização de pequenas empresas de software. Eles descobriram que as redes de relações têm um impacto sobre a seleção do mercado externo, bem como do modo de entrada no contexto de processos de rede em curso. (JOHANSON; VAHLNE, 2009, p. 1.413).

Os clusters de base tecnológica, objetos deste estudo, possuem como característica a construção de redes de cooperação, fundamentais para o processo de internacionalização das empresas neles inseridas. Para Guellec e La Poterie (2001) a internacionalização da tecnologia não é restrita a transferência da propriedade intelectual, esta amplia ainda mais para a própria geração de conhecimento. Com base na literatura prévia e nos dados coletados referente as regiões dos clusters formulou-se a seguinte hipótese:

H1: A internacionalização das empresas influencia positivamente na clusterização das regiões.

Esta hipótese busca subsidiar a primeira questão, e aponta a internacionalização como impulsionadora da inovação no cluster.

\subsection{Inovação}

A inovação é essencial para o desenvolvimento das regiões. Os polos tecnológicos são aglomerações de empresas de base tecnológica, e possuem como um de seus objetivos a construção de redes de cooperação para desenvolvimento da inovação, por meio de pesquisas e integração entre universidades, centros tecnológicos e empresas inseridas e/ou fomentadas nestes clusters.

A inovação, segundo Cassiolato e Lastres (2005) é o processo pelo qual as organizações incorporam conhecimento na produção de bens e serviços que lhe são novos, independentemente de serem novos para seus concorrentes.

Os processos de inovação são articulados nos chamados "sistema de inovação", conceituado como "[...] um conjunto de instituições distintas que contribuem para o desenvolvimento da capacidade de inovação e aprendizado de um país, região, setor ou localidade - e também o afetam". (CASSIOLATO; LASTRES, 2005, p. 37).

REAd | Porto Alegre - Edição 84 - Nº 2 - Maio / Agosto 2016 - p. 453 - 476 
Portanto, a existência de sinergia entre universidades e instituições de pesquisa e desenvolvimento (P\&D), empresas e órgãos governamentais é fundamental para a capacidade de inovação do cluster. Um indicador a ser considerado para verificar a capacidade de inovação do cluster, é o volume de submissão de patentes aos órgãos de propriedade intelectual na região. Este indicador busca subsidiar a hipótese seguinte, aportada na positividade da inovação na clusterização das regiões:

H2: A inovação influencia positivamente no grau de clusterização das regiões.

Para Schumpeter (1982) a inovação é um processo em movimento de destruição criativa no qual a empresa assimila conhecimento e gera novas ideias, produzindo novas combinações que incessantemente revolucionam a estrutura econômica destruindo a antiga e promovendo o desenvolvimento econômico.

\subsection{Clusterização e conhecimento}

Clusters podem ter um impacto econômico e social sobre a competitividade e inovação. Para Fundeanu e Badele (2014) uma abordagem de cluster aberta à inovação é dependente de pesquisas universitárias, dinamicidade do empreendedorismo, acesso a capital de risco oferecido pelo setor privado e políticas de apoio estabelecidas pelos governos. Neste sentido o conhecimento de um clusters relaciona-se de maneira mais tangível com a disponibilidade de profissionais qualificados, especialmente para o desenvolvimento em P\&D.

Os estudos de Jaffe et al. (1993) e Audretsch e Feldman (1996), inferem que transbordamentos de conhecimento costumam ser delimitados geograficamente na região clusterizada, como forma de materializar o conhecimento regional, por vezes citado em obras anteriores, utilizou-se o quantitativo do número de mestres e doutores nos clusters pesquisados para o período estudado.

Delgado, Porter e Stern (2014) inferem que clusters podem incentivar muitos dispositivos de aglomeração, incluindo acesso imediato a insumos essenciais, melhor relacionamento com clientes, melhor experimentação e inovação, mas enfatiza ainda a função do conhecimento científico local, o potencial de divulgação de conhecimentos de oportunidades de inovação e empreendedorismo. A partir desta concepção a hipótese seguinte sugere que:

H3: O nível de qualificação influencia positivamente o grau de clusterização das regiões.

REAd | Porto Alegre - Edição 84 - N² 2 - Maio / Agosto 2016 - p. 453 - 476 


\section{Fabio Lamartine Barbosa Toledo, Keller Mafioletti, Mohamed Amal \& Marianne Hoeltgebaum}

\section{METODOLOGIA}

O presente estudo trata-se de pesquisa correlacional descritiva, com abordagem quantitativa. Segundo Sampieri et al. (2006, p. 104) a pesquisa correlacional possui como objetivo " avaliar a correlação entre duas ou mais variáveis ou conceitos". Neste sentido busca-se correlacionar a internacionalização, qualificação e inovação nos clusters pesquisados.

A coleta de dados deu-se por meio de: (a) pesquisa bibliográfica nas bases de dados: Scopus, Capes, Science Direct e Web of Science; (b) pesquisa nos sites dos polos tecnológicos e das empresas incubadas ou que possuem projetos incubados nos polos; (c) consulta a base de dados de órgãos governamentais dos três países (Portugal, Espanha e Brasil) onde localizam-se os polos tecnológicos. O método quantitativo foi utilizado para o tratamento dos dados coletados, o qual de acordo com Richardson (2012, p.70) "[...] caracteriza-se pelo emprego da quantificação, tanto nas modalidades de coleta das informações, quanto no tratamento delas por meio de técnicas estatísticas $[\ldots] ”$.

Para a análise utilizou-se o software Gretl que contém uma interessante variedade de estimadores, baseados em método de momentos generalizado (GMM), possui máxima de verossimilhança e mínimos quadrados, podendo estes serem usados em modelos com uma única equação ou com sistemas de equações. Não obstante, é possível realizar análises com dados em painel, dados de corte e séries temporais. (ANDRADE, 2013).

\subsection{Modelo}

Por meio de estimações através do modelo painel, fez-se uso de dados longitudinais referente ao período de 2008 a 2011, dentre eles índice de clusterização (IC), exportações (EXP), patentes (PAT) e número de mestres e doutores (MD) de quatro regiões (Cataluña, Norte de Portugal, Recife e Florianópolis), onde localizam-se clusters tecnológicos. As dimensões contempladas pelo modelo são: internacionalização, clusterização, inovação e qualificação. Devido à dificuldade de levantamento de dados secundários de períodos anteriores e/ou posteriores, optou-se pelo recorte temporal apontado. As variáveis determinantes ao modelo proposto, poderão ser identificadas por meio da equação descrita a seguir:

$$
I C=\beta E X P_{i t}+\beta P A T_{i t}+\beta M D_{i t}+e_{i t}
$$




\section{ESTUDO EM CLUSTERS TECNOLÓGICOS IBERO AMERICANOS}

\subsubsection{Variável dependente}

A variável dependente é o índice de clusterização (IC), que foi apurado nas regiões de Portugal e Espanha, através de consulta ao Observatório Europeu de Clusters, apropriando-se de dados como o número de empresas, número de funcionários e especialização, delimitados aos seguintes setores: (a) produtos químicos; (b) educação e conhecimento de criação; (c) serviços financeiros; (d) tecnologia de informação; (e) mídia e publicação; (f) produção metalúrgica; (g) transmissão e geração de energia; (h) telecomunicações e ; (i) transportes e logística.

Em contrapartida, para as aglomerações concentradas no Brasil (Porto Digital - Recife e Certi - Florianópolis), com a finalidade de buscar as mesmas informações, foi efetuada consulta ao banco de dados estatísticos da RAIS (Relação anual de informações sociais) mantido pelo Ministério do Trabalho e Emprego, limitando-se a setores iguais ou semelhantes aos das regiões de Portugal e Espanha. Para cálculo do índice de especialização nas regiões nacionais e utilizouse a fórmula, descrita na Figura 1.
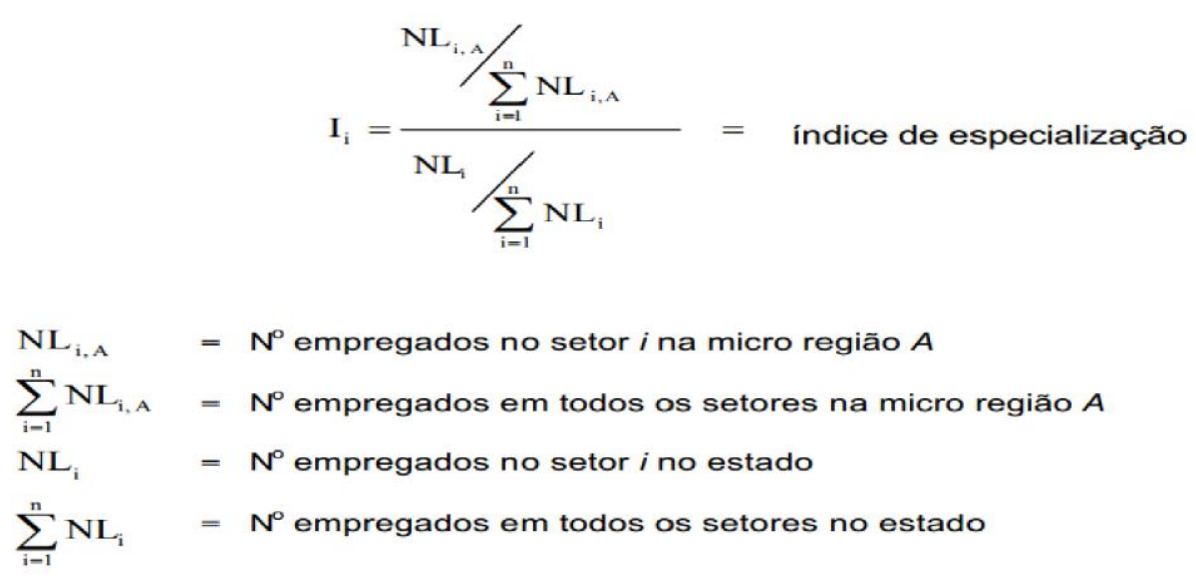

Figura 1- Cálculo do Índice de Especialização

Fonte: adaptado de Suzigan et al. (2000)

\subsubsection{Variáveis independentes}

As variáveis independentes utilizadas para subsidiar a construção do modelo sugerido nesta pesquisa, são descritas são descritas a seguir.

Exportações: As exportações podem ser consideradas como um dos fatores influentes no índice de especialização do cluster. Os clusters tecnológicos desenvolvem alta tecnologia, que está relacionada a processos rápidos de internacionalização, ou born global, empresas que nascem

REAd | Porto Alegre - Edição 84 - N² - Maio / Agosto 2016 - p. 453 - 476 


\section{Fabio Lamartine Barbosa Toledo, Keller Mafioletti, Mohamed Amal \& Marianne Hoeltgebaum}

globais. Esta relação direta entre a alta tecnologia e rápida internacionalização justifica neste estudo a utilização das exportações como uma das variáveis independentes.

Patentes: com objetivo de proteger a descoberta de um novo produto ou processo, as empresas utilizam-se do registro de marcas e patentes. As patentes protegem as invenções oriundas do resultado da investigação e desenvolvimento de novos produtos e processos e são importantes incentivos para a investigação e desenvolvimento e à inovação em geral. (OECD, 1997). Diversos estudos prévios quantificam a inovação pela mensuração do número de patentes, neste sentido optou-se pela pesquisa do registro de patentes por ser considerado como um importante indicador da inovação.

Mestre/Doutores: A qualificação pode ser considerada como um dos pilares da inovação, pois novos produtos e processos estão frequentemente relacionados ao resultado de pesquisas universitárias ou a criação de spin-off. A adoção da qualificação como uma das variáveis independentes, por meio do número de mestres e doutores, justifica-se devido a estreita relação entre os clusters tecnológicos pesquisados e as Universidades existentes nestas regiões.

\subsection{Caracterização da amostra}

A amostra foi selecionada considerando a existência de incentivo a inovação e um alto grau de especialização, por meio dos parques tecnológicos estruturados nestas regiões e sua interação com as universidades e centros tecnológicos.

O apoio governamental é outro fator semelhante em cada um destes clusters, facilitando assim a criação de entidades que auxiliam no desenvolvimento destas localidades. A seguir apresenta-se um breve histórico de organizações que fomentam cada um dos clusters de base tecnológica explorados:

\subsubsection{Fundação Centros de Referência em Tecnologias Inovadoras (CERTI)}

A Fundação Centros de Referência em Tecnologias Inovadoras (CERTI) foi fundada em 31 de outubro de 1984, na cidade de Florianópolis, Santa Catarina. Originou-se das atividades do Labmetro (Laboratório de Metrologia do Departamento de Engenharia Mecânica da UFSC Universidade Federal de Santa Catarina). Além da UFSC, participaram da formação deste centro, empresas privadas e públicas e órgãos dos governos federal e estadual. É administrada por uma superintendência e por conselhos e a partir de 1990 começa a operar em instalações próprias,

REAd | Porto Alegre - Edição 84 - N² 2 - Maio / Agosto 2016 - p. 453 - 476 
com intuito de ter uma maior amplitude em nível nacional, estabeleceu Institutos Tecnológicos em Brasília e Manaus. (CERTI, 2014).

\subsubsection{Porto Digital}

O Porto Digital, foi criado em 2000, é resultado do ambiente de inovação consolidado em Recife, capital de Pernambuco, nas últimas décadas. Em uma região atrativa para inovação, instituições, empresas, universidades e governos fomentaram mudanças econômicas e sociais que estão gerando riqueza, emprego e renda. Atualmente possuem 200 instituições entre empresas de TIC, economia criativa, serviços especializados e órgãos de fomento, que em 2010 geraram uma receita de um bilhão de reais. (Porto, 2014)

O Porto Digital é formado, em sua maioria, por pequenas e médias empresas criadas na própria cidade, mas concomitantemente abriga grandes instituições multinacionais e brasileiras. As principais áreas de competência das organizações instaladas são o desenvolvimento de sistemas de gestão empresarial, mobilidade urbana, games, animação e aplicações para dispositivos móveis, redes neurais e inteligência artificial para finance e banking, segurança de dados, e-learning, e-entertainment e outsourcing. (PORTO, 2014)

\subsubsection{Rede de Parques de Ciência e Tecnologia e Incubadoras (PortusPark)}

A Rede de Parques de Ciência e Tecnologia e Incubadoras (PortusPark), é uma organização de capitais privados e públicos, sem fins lucrativos, com estatuto de Instituição de Utilidade Pública, motivada por um conjunto relevante de entidades de apoio à competitividade e inovação, que levaram à sua constituição em 29 de Abril de 1991. (PORTUSPARK, 2014)

O conceito PortusPark baseia-se em três pontos fundamentais: i) Criação de uma Rede de Parques de Ciência e Tecnologia e de Incubadoras que cubra harmoniosamente o espaço geográfico correspondente à Região Norte de Portugal; ii) Participação nos capitais sociais dos

Parques e das Incubadoras membros da Rede; iii) Prestação de serviços avançados aos Parques de Ciência e Tecnologia e Incubadoras da Rede PortusPark, bem como às empresas instaladas na Rede. (PORTUSPARK, 2014)

2.2.4 Cluster de Tecnologia da Informação e Comunicação (TIC) Barcelona Digital

REAd | Porto Alegre - Edição 84 - N 2 - Maio / Agosto 2016 - p. 453 - 476 


\section{Fabio Lamartine Barbosa Toledo, Keller Mafioletti, Mohamed Amal \& Marianne Hoeltgebaum}

O governo catalão criou o Programa de Clusters Cataluña, iniciado em 1993, objetivando fortalecer a competitividade das empresas, fornecendo orientação estratégica, treinamento e serviços de consultoria em matéria de cooperação internacional e inovação. Alguns requisitos para que o aglomerado participe é que seja alinhado com a política que o programa tenta implementar, que seus serviços e/ou produtos tenham cobertura em toda Cataluña ao menos, que a entidade organizadora do cluster não tenha fins lucrativos, ser composto em sua maioria por empresas privadas, ter no mínimo 20 membros, tenha faturamento mínimo de 100 milhões de euros e ter um plano estratégico. (CATALUÑA, 2015)

Aproveitando tais incentivos governamentais, cria-se o cluster de TIC Barcelona Digital, nascido para subsidiar a criação de empresas, PMEs, grandes empresas e outras entidades aumentando a competitividade do sector das TIC na Cataluña, promovendo uma nova cultura empresarial baseada em colaboração e inovação aberta, registrado no Ministério da Indústria, Turismo e Comércio desde 2008, tem atualmente mais de cinquenta membros. (CLUSTERS DE CATALUNYA ANUARI, 2013)

Com o intuito de apurar indicadores de internacionalização e inovação, realizou-se levantamento do número de patentes submetidas nas respectivas regiões pesquisadas, compreendidos no período de 2008 a 2011, por meio de consulta a órgãos governamentais: Ministério do Desenvolvimento, Indústria e Comércio Exterior e o Instituto Nacional da Propriedade Industrial.

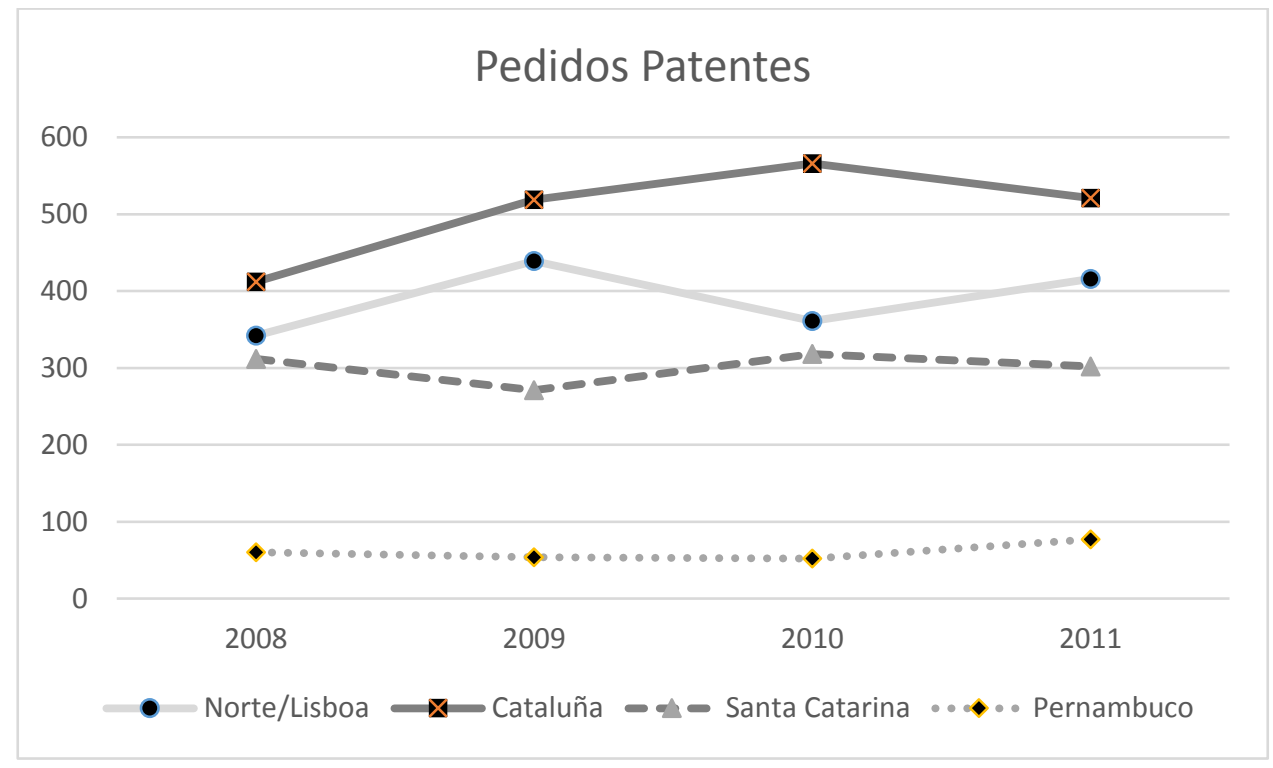

Figura 2 - Número de Patentes Submetidas

REAd | Porto Alegre - Edição 84 - N² - Maio / Agosto 2016 - p. 453 - 476 


\section{ESTUDO EM CLUSTERS TECNOLÓGICOS IBERO AMERICANOS}

Fonte: Dados da Pesquisa

Observa-se na Figura 2 que, ao menos no período selecionado, a submissão de patentes se manteve estável com pequenas oscilações, indicando a inovação como positiva para estas regiões.

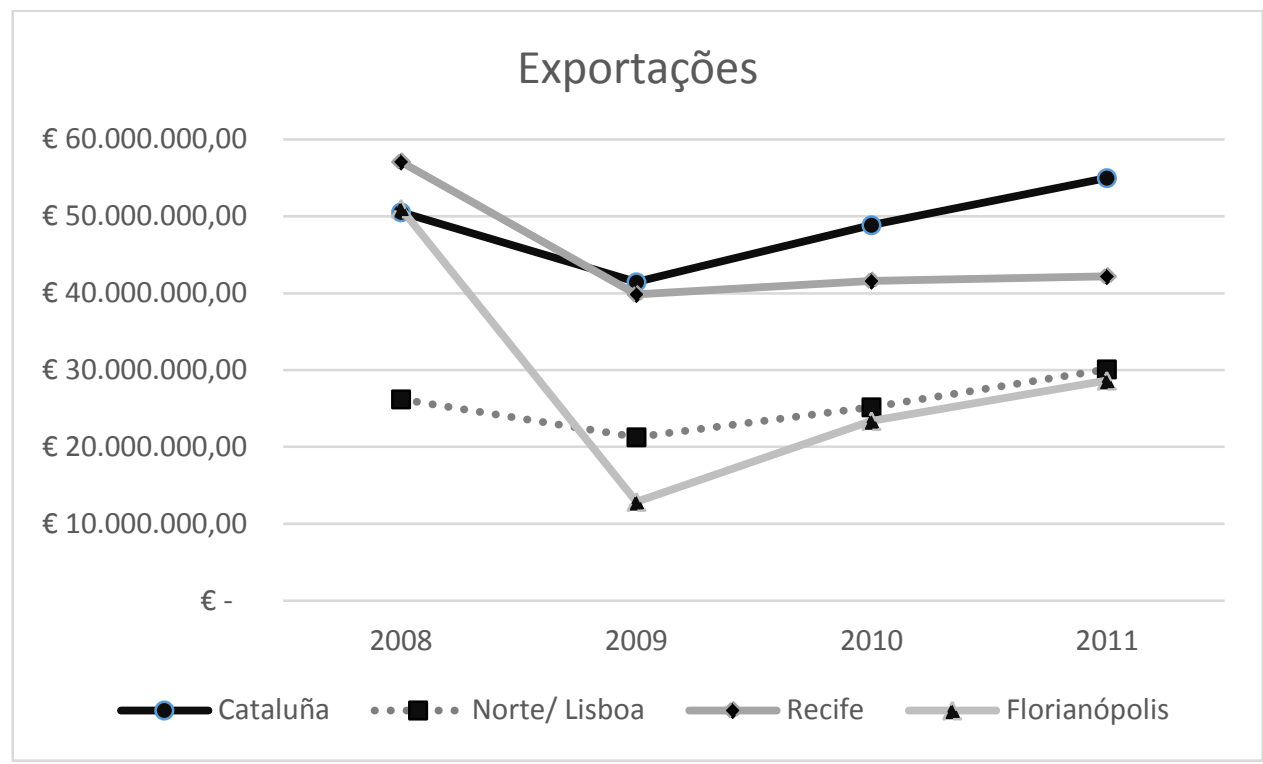

Figura 3 - Exportações em Clusters Tecnológicos Ibero-americano

Fonte: Dados da pesquisa

Almejando a apuração de um novo indicador buscou-se conhecer o volume de exportações dos clusters tecnológicos, com a finalidade de identificar seu grau de internacionalização por meio das exportações de produtos e serviços. A partir de um corte temporal de quatro anos (2008 a 2011). Com isso a Figura 3 apresenta a evolução das exportações das regiões onde localiza-se os clusters de base tecnológica pesquisados.

Observa-se quanto a este indicador, e especificamente no Brasil, foram levantadas exclusivamente da região das capitais, pois cientificados que as partes mais interioranas dos estados em tela concentram grande parte de exportações agroindustriais, isso poderia comprometer os resultados apresentados.

Ressalta-se que as unidades de moeda das exportações foram padronizadas para Euros, convertidas através do sistema do Banco Central do Brasil, com a cotação do último exercício de cada ano. Por fim, angariando a mensuração do nível de qualificação das regiões, com base em 


\section{Fabio Lamartine Barbosa Toledo, Keller Mafioletti, Mohamed Amal \& Marianne Hoeltgebaum}

sites governamentais das quatro regiões, foi possível levantar a evolução do número de mestre e doutores destas localidades.

Tabela 2 - Nível de Conhecimento do Cluster

\begin{tabular}{ccccccccc}
\hline Ano $\rightarrow$ & \multicolumn{2}{c}{$\mathbf{2 0 0 8}$} & \multicolumn{2}{c}{ 2009 } & \multicolumn{2}{c}{$\mathbf{2 0 1 0}$} & \multicolumn{2}{c}{$\mathbf{2 0 1 1}$} \\
\hline $\begin{array}{c}\text { Titulação } \rightarrow \\
\text { Região } \downarrow\end{array}$ & Mestre & Doutores & Mestre & Doutores & Mestre & Doutores & Mestre & Doutores \\
\hline Norte/Lisboa & 8189 & 974 & 14953 & 1022 & 14632 & 1110 & 15921 & 1302 \\
Cataluña & 2951 & 1615 & 4784 & 1610 & 7498 & 1716 & 8139 & 1865 \\
Pernambuco & 948 & 533 & 1096 & 673 & 1247 & 726 & 1633 & 935 \\
Santa Catarina & 997 & 390 & 1135 & 542 & 1312 & 748 & 1704 & 872 \\
\hline \multicolumn{7}{c}{ Fonte: dados da pesquisa }
\end{tabular}

O nível de conhecimento encontrado nas regiões dos clusters indica a superioridade europeia em relação aos clusters localizados no Brasil, onde possui índices de especialização e conhecimento em média $50 \%$ menor que dos clusters europeus.

\section{ESTIMAÇÃO DO MODELO E ANÁLISE}

A análise foi realizada com base na regressão de dados em painel, que considera em um mesmo modelo estatístico, dados com corte transversal de um conjunto de indivíduos "crosssection" onde esses variam e o tempo fica constante, assim como dados em séries temporais, nos quais o número de indivíduos permanece constante e o tempo varia. Ao congregar essas duas características a utilização deste método proporciona algumas vantagens, salienta-se a heterogeneidade dos indivíduos, o maior nível de informação a respeito das variáveis explicativas, menor colinearidade (podendo evitar o problema de multicolinearidade) e maior grau de liberdade para o modelo. (WOOLDRIDGE, 2001).

A estimativa através do painel dinâmico, que está sendo utilizado, requer certa ponderação. Embora o método oportunize um painel equilibrado, com todas as informações período a período, infere-se o problema de se ter apenas um curto espaço de tempo para análise. Para suprimir tal problema, a estimação através do GMM (Generalized Method of Moments) é recomendada, já que admite à inclusão de efeitos específicos e variáveis dummies temporais, demonstrando eficiência no controle sobre a endogeneidade (OZKAN, 2001).

Anteriormente a análise em painel, realizou-se a correlação dos dados, através do programa Excel, para apurar o nível de correlacionamento entre as variáveis testadas, conforme demonstrado na tabela abaixo.

REAd | Porto Alegre - Edição 84 - N² 2 - Maio / Agosto 2016 - p. 453 - 476 


\section{ESTUDO EM CLUSTERS TECNOLÓGICOS IBERO AMERICANOS}

Tabela 3 - Correlação entre variáveis.

\begin{tabular}{lcccc}
\hline & Qualificação & Patentes & Clusterização & Exportações \\
\hline Qualificação & 1 & & & \\
Patentes & 0,39383 & 1 & & \\
Clusterização & 0,81742 & $-0,00301$ & 1 & 1 \\
Exportações & $-0,27048$ & 0,45548 & $-0,32632$ & \\
\hline \multicolumn{5}{c}{ Fonte: Dados da pesquisa. }
\end{tabular}

Observa-se que as correlações entre as variáveis clusterização x patentes; clusterização x exportações; qualificação x exportações demonstram significância corrrelacional estatística negativa imperfeita, enquanto patente x qualificação; clusterização x qualificação; exportações x patentes apresenta correlação positiva imperfeita.

Com 50\% das correlações oscilando entre positiva e negativa, sendo que as positivas estão variando de moderada a forte correlação, já as negativas de moderada a fraca, então pela não prevalência de um sentido único de tendência, motivou-se a regressão em painel de todos os dados de uma única vez.

Sabendo-se que os dados em painel melhoraram certas características do modelo estimativo, porém exigindo a especificação correta de como lidar com os efeitos não observados. Tais efeitos podem ser eliminados quando a estimativa é feita por meio de efeitos fixos. A técnica complementar consiste numa estimação através de efeitos aleatórios, o que implica que os efeitos não observados não estão correlacionados com todas as variáveis independentes. A ferramenta utilizada para que o pesquisador decida entre uma forma de estimação ou outra é o Teste de Hausman, que no caso desta pesquisa demonstrou rejeição a hipótese nula, demonstrando que as estimações por mínimos quadrados ordinários (MQO) são consistentes, evidenciando que opção pela estimação através de painéis com efeitos fixos seria a mais adequada ao modelo. Então parte-se para a regressão utilizando tal recurso.

Com base em dados das regiões Ibero-americanas, cujo período foi de 2008 a 2011, apresenta-se a tabela subsequente com as respectivas estatísticas extraídas do programa Gretl.

Tabela 4 - Análise de Dados com Modelo Painel

\begin{tabular}{lccc}
\hline & Coeficiente & P-valor & Significância \\
\hline Constante & 0,598396 & $1,48 \mathrm{E}-06$ & $* * *$ \\
Qualificação & $-1,17218 \mathrm{e}-05$ & 0,257 & \\
Patentes & $-0,000367792$ & 0,0545 & $*$ \\
Exportações & $1,13 \mathrm{E}-08$ & 0,0009 & $* * *$ \\
\hline
\end{tabular}

REAd | Porto Alegre - Edição 84 - N² 2 - Maio / Agosto 2016 - p. 453 - 476 


\section{Fabio Lamartine Barbosa Toledo, Keller Mafioletti, Mohamed Amal \& Marianne} Hoeltgebaum

\begin{tabular}{lcc}
\hline $\mathrm{R}^{2}=0,97$ & Durbin-Watson: 0,97 & Estatística $\mathrm{F}=0,0006$ \\
\hline Legenda: $* * *$ Signif. a $1 \%$ & $* *$ Signif. a 5\% & $*$ Signif. a 10\% \\
\hline
\end{tabular}

Fonte: Dados da pesquisa

As estatísticas indicam significância na regressão realizada para a variável constante, patentes e exportações, fortalecidos pela estatística $\mathrm{R}^{2}$, que foi superior a $97 \%$, demonstrando que as variações de y (variável dependente), estão sendo suficientemente explicadas pelas variações de x (variáveis independentes), apenas a variável qualificação não apresenta significância ao modelo, nem mesmo ao ser realizada tentativa de regressão desta variável separadamente com as variáveis patentes e clusterização, a fim de confirmação da hipótese 3, o resultado foi análogo ao anterior.

E ainda, através do teste de estatística F, foi possível observar um baixo valor, rejeitando-se novamente a hipótese nula, e ainda para o teste de normalidade dos resíduos constatou-se distribuição normal de erro. Foram utilizados testes de erro padrão robustos e inseridas variáveis dummies, elemento importante para analise econométrica, usual em estudos de caráter espacial, temporal ou qualitativos (Rebelo \& Valle, 2002).

Denota-se que os clusters aqui explorados tenham se proliferado as margens de representativas universidades. Embora a literatura afirme que a transmissão do conhecimento através de parcerias entre universidades e indústria, sejam fatores de relevância para o desenvolvimento regional (ETZKOVITZ, 2012), e ainda que o transbordamento de conhecimento tem representatividade em áreas clusterizadas (JAFFE et al, 1993; AUDRETSCH; FELDMAN, 1996) a variável qualificação, extraída a partir da evolução do quantitativo de mestres e doutores das regiões não foi significativo ao modelo. Sugerindo que ao menos nestas regiões delimitadas, a evolução deste indicador não é fator preponderante e explicativo ao modelo.

Enquanto as outras duas variáveis medidas patentes e exportações, mostraram relevância estatística. Sendo que este resultado, está de acordo ao já previsto pela literatura que correlacionou cluster, inovação e internacionalização. (DELGADO; PORTER; STERN, 2014; LIBAERS; MEYER, 2011; FUNDEANU; BADELE, 2014;). Em especial as exportações se mostraram os preditores mais sensíveis à clusterização dado ao alto de nível de significância alcançado na estimação. Então, sugere-se que o agrupamento confere vantagem comparativa as regiões, facilitando o comercio internacional (ARCANGELIS; FERRI; PADOAN, 2002).

REAd | Porto Alegre - Edição 84 - N² - Maio / Agosto 2016 - p. 453 - 476 


\section{ESTUDO EM CLUSTERS TECNOLÓGICOS IBERO AMERICANOS}

Mesmo havendo diferenças culturais relevantes nas quatro localidades exploradas, as teorias aqui apresentadas se mostram válidas em sua maioria. $\mathrm{O}$ advento da globalização como propulsor para empresas buscarem outros mercados, que outrora forçava grandes empresas a se multinacionalizar, atualmente demonstra que a aglomeração industrial articulada nas localidades, muitas vezes aporta melhores benefícios que a expansão física e internacional das organizações. Especificamente neste caso, permite-se inferir que vantagens como a inovação através de patentes de novos produtos (DELGADO; PORTER; STERN, 2014) ou facilidades de adimplemento de exportações (KAFOUROS et al., 2008), influencia para que regiões se clusterizem de maneira crescente.

A seguir observa-se uma tabela com os resultados finais apurados pela pesquisa, com apoiados nos métodos anteriormente descritos.

Tabela 5 - Resultados Finais

\begin{tabular}{lc}
\hline HIPÓTESES & RESULTADO \\
\hline H1: A internacionalização das empresas influencia positivamente na clusterização das regiões. & Suportada \\
H2: A inovação influencia positivamente no grau de clusterização das regiões. & Suportada \\
H3: O nível de qualificação influencia positivamente no grau de clusterização das regiões. & Não há suporte
\end{tabular}

Fonte: Dados da pesquisa.

A hipótese 1, a qual sugeria que a internacionalização das empresas influencia positivamente na clusterização das regiões, foi confirmada, em concordância com as pesquisas de Arcangelis, Ferri e Padoan (2002).

Constatou-se que a inovação influencia no grau de clusterização das regiões, resultado que está em consonância com o encontrado por Rodríguez e Valencia (2008), respondendo assim a segunda questão da pesquisa e suportando a hipótese número 2. Por fim, a hipótese 3, a qual inferia que a qualificação de uma região influencia na clusterização da região, ao menos nas regiões estudadas, não foi confirmada.

\section{CONSIDERAÇÕES FINAIS}

Estudos comparativos entre clusters de vários países, são bastante válidos para fins de verificação, confrontação, propagação e generalização de conceitos, que nem sempre são expansíveis a todas as partes do mundo, frisa-se como exemplo, o caso muito particular do desenvolvimento dos distritos industriais da terceira Itália.

REAd | Porto Alegre - Edição 84 - N² - Maio / Agosto 2016 - p. 453 - 476 


\section{Fabio Lamartine Barbosa Toledo, Keller Mafioletti, Mohamed Amal \& Marianne Hoeltgebaum}

Neste sentido, este estudo objetivou por meio da comparação entre os clusters de Portugal, Brasil e Espanha, responder as questões: Em que medida o grau de especialização depende do volume de exportações das empresas inseridas nestes clusters? Qual o efeito da inovação sobre o grau de especialização destas regiões? Quanto maior for o nível de conhecimento do cluster (qualificação profissional) maior será o efeito da inovação sobre a especialização?

A primeira pergunta é respondida por meio da confirmação da primeira hipótese, afirmando assim a influência da internacionalização na clusterização das regiões. Da mesma forma a segunda questão, onde a inovação se mostrou influente ao índice de clusterização, contudo pelo Brasil não ser representativo na geração de patentes, possivelmente as inovações não puderam ser captadas integralmente por este indicador, gerando assim menos significância e este que as exportações. Por fim contatou-se resposta negativa à terceira questão, ancorada pela terceira hipótese, que demostrou não haver suporte para tal afirmação, possivelmente pela discrepância entre o número de Mestres e Doutores do Brasil perante aos países europeus, o que não demonstrou convergência entre eles, ou seja, a discriminação deste indicador entre os clusters é baixa.

Conclui-se que ocorreu inter-relação entre certas variáveis da pesquisa, integral ou parcialmente, mantendo compatibilidade com a teoria, demonstrando que um cluster é fértil a inovação e internacionalização, porém a qualificação não comprovou ser característica com influência eminente neste modelo.

Conforme já esperado, os clusters da Espanha e de Portugal possuem maior número de mestres e doutores, que provavelmente implicaram no maior índice de inovação, comprovados pelo número de patentes. Não sendo possível observar este mesmo efeito em Pernambuco, que embora com números de qualificação superiores aos de Santa Catarina e com índices de clusterização similares, possuem uma menor produtividade quanto ao patenteamento, não conseguindo acompanhar os países europeus, não corroborando para que a teoria se mostre válida. Embora o estudo buscasse por regiões clusterizadas relativamente homogenias, diferenças com patenteamento e qualificação se mostraram gritantes, recomendando-se investimentos nestas áreas em prol de uma evolução no setor.

Após a pesquisa, sugere-se que com a disponibilização dos dados, futuras pesquisas sejam realizadas, pois no ano de 2015, somente os dados até 2011 estão disponíveis para consulta, considerando que o Brasil foi o país com maior dificuldade de acesso às informações necessárias.

REAd | Porto Alegre - Edição 84 - Nº 2 - Maio / Agosto 2016 - p. 453 - 476 


\section{ESTUDO EM CLUSTERS TECNOLÓGICOS IBERO AMERICANOS}

Há também possibilidade de novas pesquisas aportarem cortes temporais mais longilíneos, assim como realizar um estudo com empresas de cada região apurando as mesmas dimensões, sugere-se ainda a utilização de outros recursos estatísticos, e infere-se, ao se tratar de estudos em países em desenvolvimento, como fundamental a utilização de novas variáveis, como por exemplo P \& D das empresas, para capturar o efeito da inovação nas localidades.

Considera-se importante que outras relações sejam verificadas, objetivando-se conhecer os impactos da localização geográfica do cluster. E embora este trabalho tenha tratado de inovação e conhecimento como influentes à clusterização não explorou lacunas dos instrumentos influenciadores à estes agentes, apontados por Autant-Bernard et al. (2013), como: observatórios regionais de inovação, plataformas locais de disseminação de conhecimento, projetos de polos científicos de excelências, podendo ser abordados em pesquisas futuras, nestas localidades ou em outras distintas.

\section{REFERÊNCIAS}

ARCANGELIS, G; FERRI, G.; PADOAN, P. C. Firms' Clustering and South-EasternEurope Export Performance: Lessons from the Italian Experience, 2002.

ANDRADE, C. H. C. de. Manual de Introdução ao Pacote Econométrico Gretl. Artigo PPGE/UFRGS, RS - 2013.

AUDRETSCH, D. B. FELDMAN, M.P. Spillovers de I \& D e da geografia da Inovação e Produção. American Economic Review, p. 630-640, 1996.

AUTANT-BERNARD, C.; FADAIRO, M. F.; MASSARDA, N. Knowledge diffusion and innovation policies within the European regions: Challenges based on recent empirical evidence. Research Policy. v.42, p. 196-210, 2013.

BAPTISTA, R.; SWANN, P. Do firms in clusters innovate more? Research Policy, v. 27, p. 525-540, 1998.

BECCHETTI, L.; ROSSI, S. The Positive Effect of Industrial District on the Export Performance of Italian Firms. Review of Industrial Organization, v. 16, n. 1, p. 53-68, 2000.

BELSO-MARTÍNEZ, J. A. Do Industrial Districts influence export performance and export intensity? Evidence for Spanish SMEs' internationalization process. European Planning Studies, v. 14, n. 6, p. 791-810, 2006.

BRENNAN, L., GARVEY, D. The role of knowledge in internationalization. Research in International Business and Finance, v. 23, p. 120-133, 2009.

REAd | Porto Alegre - Edição 84 - N² 2 - Maio / Agosto 2016 - p. 453 - 476 
BREITZMAN, A.; THOMAS, P. The Emerging Clusters Model: A tool for identifying emergingtechnologies across multiple patent systems. Research Policy, v. 44, p. 195-205, 2015.

BRASIL. Capes - Geocapes Sitemas de Informações Georreferenciadas. Disponível em: <http://geocapes.capes.gov.br/geocapes2>. Acesso em: 28 dez. 2014.

BRASIL. Instituto Nacional de Propriedade Intelectual Disponível em: < http://www.inpi.gov.br/portal/artigo/estatisticas> Acesso em $28 \mathrm{dez} 2014$.

BRASIL. Ministério do Desenvolvimento, Industria e Comercio Exterior. Disponível em <http://www.mdic.gov.br//sitio/interna/index.php?area=5>. Acesso em: 23 dez. 2014.

BRASIL. Ministério do Trabalho e Emprego - RAIS Disponível em http://bi.mte.gov.br/bgproger/login.php>. Acesso em: 03 jan. 2015.

CARLSSON, B. Internationalization of innovation systems: A survey of the literature. Research Policy. v.35, p. 56-67, 2006.

CASSIOLATO, J. E.; LASTRES, H.M.M. O foco em arranjos produtivos e inovativos locais de micro e pequenas empresas. In: LASTRES, H.M.M.; CASSIOLATO, J.E.; MACIEL, M.L. (Orgs.) Pequena Empresa: Cooperação e Desenvolvimento Local. Rio de Janeiro: Relume Dumará, 2003.

Sistemas de inovação e desenvolvimento: as implicações de política. São Paulo em perspectiva, v. 19, n. 1, p. 34-45, jan./mar. 2005.

CLUSTERS DE CATALUNYA, ANUARI, 2013, Generalitat de Catalunya, Departament d'Empresa i Ocupació, Direcció General d'Indústria, Raül Blanco Díaz (Coordinador), Barcelona, p. 106-109, jun- 2014.

CLUSTER OBSERVATORY. Disponível em: 〈http://www.clusterobservatoy.eu> Acesso em: 20 dez. 2014.

DELGADO, M.; PORTER, M.; SCOTT, S. Clusters, convergence, and economic. Research Policy. v. 43, p. 1785-1799, 2014.

DIEZ-VIAL, I.; FERNÁNDEZ-OLMOS, M. Moderating influence of internal resources on cluster externalities. EuroMed Journal of Business, v. 9, n. 1, p. 75-92, 2014.

ECOMMERCE \& TECH BARCELONA. Disponível em: 〈http://www.ecommercetechbcn.com $>$ Acesso em 27 dez. 2014.

ETZKOWITZ, H. The norms of entrepreneurial science: cognitive effects of the new universityindustry linkages. Research policy, v. 27, n. 8, p. 823-833, 1998. 


\section{ESTUDO EM CLUSTERS TECNOLÓGICOS IBERO AMERICANOS}

ETZKOWITZ, H. Triple helix clusters: boundary permeability at university-industrygovernment interfaces as a regional innovation strategy. Environment and Planning-Part $\mathbf{C}$, v. 30, n. 5, p. 766, 2012.

FERNHABER, S. A.; GILBERT, B. A.; MCDOUGALL, P. P. International entrepreneurship and geographic location: An empirical examination of new venture internationalization. Journal of International Business Studies, v. 39, p. 267-290, 2008.

FUNDEANU, D. D.; BADELE, C. S. The impact of regional innovative clusters on competitiveness. Procedia - Social and Behavioral Sciences, v. 124, p. 405 - 414, 2014.

FUNDAÇÃO CERTI. Disponível em: < http://www.certi.org.br/pt/acerti-historico > Acesso em: 24 dez 2014.

GENERALITAT DE CATALUNYA - INSTITUT D'ESTATISTÍCA DE CATALUNYA. Disponível em <http://www.idescat.cat/pub/?id=aec\&n=375\&t=2012\&x=7\&y=8\&lang=es $>$. Acesso em: 22 dez. 2014.

GEORGE, G.; ZAHRA, S.; WOOD, R. D. The effects of business-university alliances on innovative output and financial performance: a study of publicly traded biotechnology companies. Journal of Business Venturing, v. 17, p. 577-609, 2002.

GUELLEC, D., LA POTTERIE, B. V. P.The internationalisation of technology analysed with patent data. Research Policy, v. 30, p. 1253-1266, 2001.

JAFFE, A.; TRAJTENBERG, M.; HENDERSON, R. Geographic localization of knowledge spillovers as evidenced by patent citations. Quarterly Journal of Economics, v. 63, p. 677-598, 1993.

KAFOUROS et al. The role of internationalization in explaining innovation performance. Technovation, v. 28, p. 63-74, 2008.

KNIGHT, G.A.; CAVUSGIL, S.T. The Born Global Firm: A Challenge to Traditional Internationalization Theory. Advances in International Marketing. v. 8, p. 11-26, 1996.

LAI, Y. et al. The effects of industry cluster knowledge management on innovation performance. Journal of Business Research, v. 67, p. 734-739, 2014.

LIBAERS, D.; MEYER, M. Highly innovative small technology firms, industrial clusters and firm internationalization. Research Policy, v. 40, p. 1426- 1437, 2011.

MACCARINI, M. E.; SCABINI, P.; ZUCCHELLA, A. Internationalization strategies in Italian district-based firms: theoretical modeling and empirical evidence. In: Clusters, Industrial Districts and Firms: the Challenge of Globalization. Conference in honour of Professor Sebastiano Brusco. 2003. Anais... Modena, 2003.

REAd | Porto Alegre - Edição 84 - N² 2 - Maio / Agosto 2016 - p. 453 - 476 
Fabio Lamartine Barbosa Toledo, Keller Mafioletti, Mohamed Amal \& Marianne Hoeltgebaum

MARSHAlL, A. (1890) Princípios de Economia. São Paulo: Nova Cultural, 1983.

MARTÍNEZ, A.; BELSO-MARTÍNEZ, J. A.; MÁS-VERDÚ, F. Industrial clusters in Mexico and Spain: Comparing inter-organizational structures within context of change. Journal of Organizational Change Management. v. 25, n. 5, p. 657-681, 2012.

OZKAN, A. Determinants of capital structure and adjustments to long run target: evidence from UK company panel data. Journal of Business Finance and Accounting. Jan/Mar 2001, pp. 175 $-199$.

PORDATA - BASE DE DADOS PORTUGAL CONTEMPORÂNEO. Disponível em <http://www.pordata.pt/Municipios/Ambiente+de+Consulta/Tabela $>$. Acesso em: 20 dez. 2014.

PORTO DIGITAL. Disponível em: <http://www2.portodigital.org/> Acesso em 25 de dezembro de 2014.

PORTER, M. E. Clusters and the new economics of competition. Harvard Business Review, v.76, n. p. 77-90 nov./dec., 1998.

. Competição: estratégias competitivas essenciais. Rio de Janeiro: Campus, 1999.

PORTUGAL. Direção-geral de estatísticas da educação e ciência. Estatisticas Oficiais. Disponível em:

$<$ http://www.dgeec.mec.pt/np4/EstatDiplomados/\%7B \$clientServletPath\%7D/?newsId=132\&fil eName=Diplomados_2000_01_2007_08.pdf > . Acesso em: 21 dez. 2014.

PORTUGAL. Instituto Nacional de Estatística - Statistics Portugal. Disponível em: < http://www.ine.pt/xportal/xmain?xpid=INE\&xpgid=ine_main >. Acesso em: $29 \mathrm{dez} 2014$.

PORTUGAL. Instituto Nacional de Propriedade Intelectual. Disponível em: <http://www.marcasepatentes.pt/index.php?section=315>. Acesso em: 27 dez. 2014.

PORTUSPARK. Disponível em <http://www.portuspark.org/> Acesso em 26 de dezembro de 2014.

REBELO, E; VALLE, P.O. O uso de regressores dummy na especificação de modelos com parâmetros Variáveis. Revista de Estatística, $3^{\circ}$ quadrimestre de 2002, pp. 17-40.

RICHARDSON, R. J. et al. Pesquisa social: métodos e técnicas. 3.ed. São Paulo: Atlas, 2012.

RODRÍGUEZ, F. B.; VALENCIA, J. C. N. La innovación tecnológica en el contexto de los clusters regionales. Cuadernos de Administración, v. 21, n. 37, p. 133-159, 2008.

ROPER, S.; LOVE, J. H. Innovation and export performance: evidence from the UK and German manufacturing plants. Research Policy, v. 31, p. 1087-1102, 2002.

REAd | Porto Alegre - Edição 84 - N² - Maio / Agosto 2016 - p. 453 - 476 


\section{ESTUDO EM CLUSTERS TECNOLÓGICOS IBERO AMERICANOS}

SAMPIERI, R. H.; COLLADO, C. H.; LUCIO, P. B. Metodologia de pesquisa. 3. ed. São Paulo: McGraw-Hill, 2006.

SCHMITZ, H. Collective efficiency and increasing returns. Cambridge Journal of Economics, Oxford, v. 23, n. 4, p. 465-483, jul. 1999.

SCHUMPETER, J. A. Teoria do Desenvolvimento Econômico: uma investigação sobre lucros, capital, crédito, juro e o ciclo econômico. São Paulo: Abril Cultural, 1982.

SUZIGAN, Wilson et al. Aglomerações industriais no Estado de São Paulo. XXVIII Encontro Nacional de Economia, 28, 2000, Anais... Campinas: ANPEC, 2000.

TRISTÃO, H. M. et al. Innovation in industrial clusters: a survey of footwear companies in Brazil. Journal of Technology Management \& Innovation. v. 8, n. 3, p. 45-56, 2013.

WOOLDRIDGE, J. M. Econometric analysis of cross section and panel data. MIT press, 2001.

WEI J.; LI-RAN, L.; XUE-MEI, X. Diffusion of technical innovation based on industryuniversity-institute cooperation in industrial clusters. The Journal of China Universities of Posts and Telecommunications, v. 17, p. 45-50, dec. 2010.

ZAHRA, S. A.; GEORGE, G. International entrepreneurship: the current status of the field and future research agenda. In: HITT, M. A. et al. (Ed.). Strategic entrepreneurship: creating an integrated mindset. Oxford: Blackwell, 2002.

ZAHRA, S.; GARVIS, D. International Corporate Entrepreneurship and Company Performance: The Moderating Effect of International Environmental Hostility. Journal of Business

Venturing. v. 15, p. 469-492, 2000. 